Review

\title{
The use of Probiotics in Horses with Gastrointestinal Disease
}

\author{
${ }^{1}$ Jelena Gotić, ${ }^{1}$ Darko Grden, ${ }^{2}$ Nikica Prvanović Babić and ${ }^{1}$ Vladimir Mrljak \\ ${ }^{I}$ Clinic for Internal Diseases, Faculty of Veterinary Medicine, University of Zagreb, Croatia \\ ${ }^{2}$ Clinic for Obstetrics and Reproduction, Faculty of Veterinary Medicine, University of Zagreb, Croatia
}

\author{
Article history \\ Received: 29-03-2017 \\ Revised: 26-07-2017 \\ Accepted: 25-08-2017 \\ Corresponding Author: \\ Jelena Gotić \\ Clinic for Internal Diseases, \\ Faculty of Veterinary Medicine, \\ University of Zagreb, Croatia \\ E-mail: jselanec@vef.hr
}

\begin{abstract}
Probiotics are common adjunctive therapy used in human and veterinary medicine, mostly intended for gastrointestinal diseases treatment or prevention. Lactic acid bacteria: Lactobacilli, bifid bacteria and enterococci and Saccharomyces boulardii, a subtype of the nonpathogenic yeast have been most frequently used as probiotic active ingredients. To become a recognized effective probiotic, a certain microorganism must fulfill many expectations, primarily the ability to survive gastrointestinal tract passage, adhere to epithelial cells and colonize intestines to present antimicrobial effect and inhibition of enteric pathogens. Prior to evaluation of clinical efficiency, it is advised to perform in vitro studies in order to identify potentially beneficial properties of probiotic organisms. An effective probiotic product could be of a great value in equine medicine, since both, adult horses and foals, frequently exhibit gastrointestinal problems. However, very little objective research has been carried out on the topic. The effectiveness of probiotic products in horses has been proven in very few studies and some presented serious adverse effects. Therefore, the benefits of probiotics use in equine medicine still remain undetermined.
\end{abstract}

Keywords: Probiotics, Equine, Gastrointestinal Tract, Microbiota

\section{Introduction}

Probiotics have become an attractive alternative or addition to usual therapies in both, human and veterinary medicine. Easily available, inexpensive and generally regarded harmless, they are often used as an adjunctive therapy in horses, to prevent or treat gastrointestinal diseases (Weese, 2003).

Metchnikoff (1908) was the first to describe the potential effects of probiotics, noticing the link between the longevity of certain ethnic groups and their diet (fermented milk products). The author supposed that these products changed the intestinal microflora keeping a good balance between pathogenic and nonpathogenic bacteria and he defined probiotics as "live microorganisms, which exhibit a health promoting effect". The term probiotic, as an antonym to antibiotic, was initially used to describe substances favoring the growth of microorganisms (Lilley and Stillwell, 1965). World Health Organization currently defines probiotics as "live (nonpathogenic) microorganisms that confer a health benefit to the host when administered in adequate amounts" (Schlundt, 2014).

The term probiotic covers a great variety of microorganisms and is therefore inaccurate to ascribe the same characteristic to all probiotics "just as all antibiotics are not effective against all bacterial infections, all probiotics will not be effective for all conditions" (Weese, 2001). A lot is still unknown about the benefits and the mechanism of action or adverse effects, due to the general "paucity of objective research in veterinary medicine" (Weese, 2001). Not surprisingly, probiotic treatment is therefore approached with skepticism by many authors (Berg, 1998; Weese, 2001; Weese and Martin, 2011).

Most of the research on probiotic effects in human medicine suggests a range of potential health benefits, which could be used in veterinary patients as well (Wynn, 2009). Among many validated useful characteristics, research most commonly acknowledges positive aspects in the treatment of acute and chronic diarrhea in children and adults (Isolauri et al., 1991; Guandalini et al., 2000; Gupta et al., 2000; Saavedra, 2000).

Manipulation of the ecology of the gastrointestinal tract has powerful systemic effects (Wynn, 2009) and probiotics have, hence, found a significant place in food animal production (Zhao et al., 1998; Van Immerseel et al., 2006; Lallès et al., 2007). Used to prevent economic losses, probiotics help decrease shedding of enteropathogenic bacteria, such as Salmonella spp and enterotoxigenic Escherichia coli and possibly reduce symptoms and 
duration of diarrhea (Zhao et al., 1998; Kyriakis et al., 1999; Van Immerseel et al., 2006). Use of probiotics clearly enhances immune function and improves the outcome of the treatment of dogs and cats with certain gastrointestinal conditions. Particularly, studies in dogs and cats have proven several positive effects of probiotics on inflammatory bowel disease, diarrhea, pancreatitis and chronic renal disease (Wynn, 2009).

In this review we gathered the available studies on various probiotic effects in equine medicine, in order to obtain a clearer picture of their use in gastrointestinal disorders.

\section{Probiotics}

Most commonly used probiotic products are Lactic Acid Bacteria (LAB), mainly lactobacilli, bifidobacteria and enterococci. Saccharomyces boulardii, a subtype of the nonpathogenic yeast Saccharomyces cerevisiae, is a yeast strain most commonly used and investigated for its probiotic properties (Fuller, 1991; Bleichner et al., 1997; Zbinden et al., 1999; Furr, 2014). Although their probiotic effects in horses are frequently observed, most yeasts act only as nutritional supplements (Weese, 2002a).

Choosing a proper microorganism for probiotic use can be difficult, since probiotic properties are not demonstrated by all probiotic microorganisms. Even different strains of the same bacterial species, same bacteria in different species, or same animal species of different age, may not show the same beneficial probiotic effects (Gibson and Fuller, 2000; Kailasapathy and Chin, 2000; Weese, 2003). Therefore, all the potential probiotic organisms should be tested for their in vitro beneficial probiotic properties prior to in vivo studies (Gorbach, 2000; Kailasapathy and Chin, 2000; Weese et al., 2003). Some authors (Gibson and Fuller, 2000; Saarela et al., 2000) favor host species-specific probiotics, although across-species efficacy is proved for some probiotics (Lee et al., 2000). Others (Weese et al., 2004; Schoster et al., 2014) consider that probiotic strains should be selected based on their probiotic properties tested in vitro and not their origin.

Every probiotic should possess some essential properties, which include characteristics that enable them to survive the passage through the gastrointestinal tract (Gibson and Fuller, 2000; Kailasapathy and Chin, 2000). The latter includes surviving acid and bile, which they would encounter in stomach and small intestine. Adhering to intestinal epithelial cells and colonization of the intestinal tract for some period is another desirable property. Moreover, producing antimicrobial factors and enteric pathogens inhibition may be critical for prevention or treatment of certain diseases. Other desirable properties of probiotics also include immune modulation, metabolic activities modulation and procarcinogens inactivation (Gibson and Fuller, 2000; Gorbach, 2000). At the end, probiotic organisms should not exhibit adverse effects, even when given at very high doses (Kailasapathy and Chin, 2000).

\section{Quality and Safety of Probiotic Preparations}

Several serious concerns regarding probiotic products are encountered due to quality issues, in both human and veterinary medicine (Hamilton-Miller and Shah, 2002; Weese, 2002b). Since they are considered food supplements, they do not undergo strict regulatory oversight (Reid, 2000), allowing for various products of poor quality to be put on the market. The research on veterinary and human commercial probiotic products showed some alarming results. A recent study evaluating veterinary preparations showed only $0.08 \%(2 / 25)$ of products to be acceptable, when considering labeling and content (Weese and Martin, 2011). Another study found only $43 \%$ of human products and $8 \%$ of veterinary products adequately labeled. Most had inadequate descriptions of the bacterial content, i.e. missing names, unspecified strains, spelling errors, nonexistent or incorrect names (Weese, 2003). Therefore, Weese (2003) proposed that every probiotic product label should declare the probiotic organisms to the strain level, spell them correctly and state the number of live organisms. However, very low levels of viable organisms, obviously less than claimed by the label and products containing other species of organisms, were commonly reported (Hamilton-Miller and Shah, 2002; Weese, 2002b; Weese and Martin, 2011). Inadequate content of commercial probiotic formulations makes their efficacy unpredictable (Schoster et al., 2014). These studies emphasize the caution in commercial probiotic product selection, but also the importance of bacteria viability control in research studies. When purchasing and using a probiotic product most horse owners and veterinarians believe the worst consequence of using them is their ineffectiveness (Schoster et al., 2014). However, adverse effects of probiotic administration in horses have been reported in experimental studies, although their incidence was not recorded in retrospective studies (Ward et al., 2004; Weese and Rousseau, 2005; Schoster et al., 2015a). Particularly, a study by Ward et al. (2004) described a case of hives after administration of probiotic preparation in an adult horse. Two other clinical studies reported development of diarrhea in foals given probiotic products (Weese and Rousseau, 2005; Schoster et al., 2015a). The number of reports on adverse effects in probiotic use in horses has to be observed considering little objective studies done on the topic.

Some serious concerns have been raised, supporting the hypothesis that typical probiotic genera can act as potential reservoirs of resistance for food or gut pathogens (Gueimonde et al., 2013; Schoster et al., 2014). Most of the available data on antibiotic resistance 
are available for lactobacilli, which have been proven to transfer genes to different gram-positive bacteria, but also acquire resistance genes from them (Mater et al., 2008). Due to their potential pathogenicity, antimicrobial resistance for clinically relevant antibiotics and in vitro evidence of enhancing adhesion of enteropathogens, enterococci also represent a serious safety issue and have, therefore, been avoided in studies considering potential probiotics in animals (Lund and Edlund, 2001; Rinkinen et al., 2003; Weese et al., 2004; Cebrian et al., 2012; Gueimonde et al., 2013).

Unwanted aspects of probiotic use were detected when a sterile bacterial supernatant of one LAB, a strain of L. salivarius, increased in vitro growth of Salmonella and Escherichia coli. The same process in vivo could cause great damage, another confirmation of the necessity for in vitro testing (Weese et al., 2004).

\section{Probiotics in Equine Medicine}

An effective probiotic product could be tremendously valuable in equine medicine, since horses frequently exhibit gastrointestinal problems (Fig. 1). Unnatural living conditions for most horses represent the greatest risk to their gastrointestinal health. Unabeling free access to grass and hay and overfeeding with rapidly fermenting carbohydrates exposes horses to colics or laminitis more then in open pasture conditions (Kronfeld and Harris, 1997). Due to its' sensitivity, specifics of equine gastrointestinal tract should be considered when choosing an appropriate probiotic preparation (Weese et al., 2004; Schoster et al., 2015b). In horses the treatment of the gastrointestinal tract by the recovery of microbiota may represent "the next frontier in equine gastroenterology" (Mullen et al., 2016).

The large intestine of a horse is inhabited by diverse microbiota consisting of bacteria, protozoa and fungi (Weese et al., 2004; Al Jassim and Andrews, 2009; Schoster et al., 2013). Research on horses is crucial in determining the exact microorganisms that work as the core microbiota, keeping a balance against enteropathogens (Mullen et al., 2016). Every horse has a unique range of microorganisms acting as their microbiota, affecting the function of the digestive system, immune stimulation, pathogen protection and metabolism. Large variations of microbial populations along the gastrointestinal tract were present even in horses living in similar dietary and husbandry conditions (Schoster et al., 2013). The complex intestinal microbiota of healthy horses is dominated by the bacteria phylum Firmicutes, with 46-70\% of identified 16S rRNA sequences in equine feces (Costa et al., 2012). This phylum includes the vast Clostridia class, containing various genera associated with gut health. Those could theoretically achieve better therapeutic modification of the microbiota (Schoster et al., 2013). An interesting finding, suggesting the importance of the Clostridiales order in the equine digestive tract proved their greater abundance in healthy as compared to diseased horses as Costa et al. (2012) found genera Trepidimicrobium and Clostridium to be more frequently determined in healthy horses $(11 \%$ of sequences) in comparison to horses with colitis $(5.5 \%)$. The same study found no difference in the Lactobacillales order, between healthy and diseased horses. The focus of probiotics studies in horses should be on the species with a greater influence on the gastrointestinal health (Schoster et al., 2014). Gastrointestinal dysbiosis is thought to be an important cause of various types of diseases in horses, emphasizing the potential therapeutic effect of gastrointestinal microbiota manipulation (Mullen et al., 2016).

The natural colonization of the gastrointestinal tract in horses begins when foals, 2-5 weeks old, eat their own dams manure (Francis-Smith and Wood-Gush, 1977). This may suggests probiotics therapy should have great success in very young foals. However most of the studies done in foals of that age presented disappointing results, instead of preventing diarrhea, foals receiving probiotics were more likely to develop diarrhea, depression, anorexia and colic, requiring additional veterinary intervention (Weese and Rousseau, 2005; Schoster et al., 2015a; 2015b).

\section{Clinical Indications for Probiotic use in Horses}

Various conditions have been proven to alter equine intestinal or fecal microbiota; colitis and colic, grass sickness, laminitis, antimicrobial administration and diet changes are just some of many (Garrett et al., 2002; Harlow et al., 2013; Costa et al., 2015a; 2015b). The changes occurring in the digestive tract of sick animals can be immense and understanding the impact the disease has on microbiota is essential (Costa and Weese, 2012; Mullen et al., 2016). However, it is questionable whether administration of the currently available probiotics, with a limited number of bacterial strains, could have any effect whatsoever on the gastrointestinal disorders in horses (Schoster et al., 2014).

Prophylactic use of probiotics could be helpful during times of high risk for the diarrhea development (diet changes, transportation, veterinary hospitals stay, minor surgical procedures, etc.). There is, however, a big difference in therapeutic pursuits in healthy animals in comparison to sick ones. Probiotics are needed mostly when disease occurs, but administering probiotic organisms together with other drugs can affect their survival in the gastrointestinal tract and make them completely ineffective (Weese, 2002a). That is why special attention should be paid on treatments requiring both, antibiotic and probiotic use, since they should never be administered concurrently. 


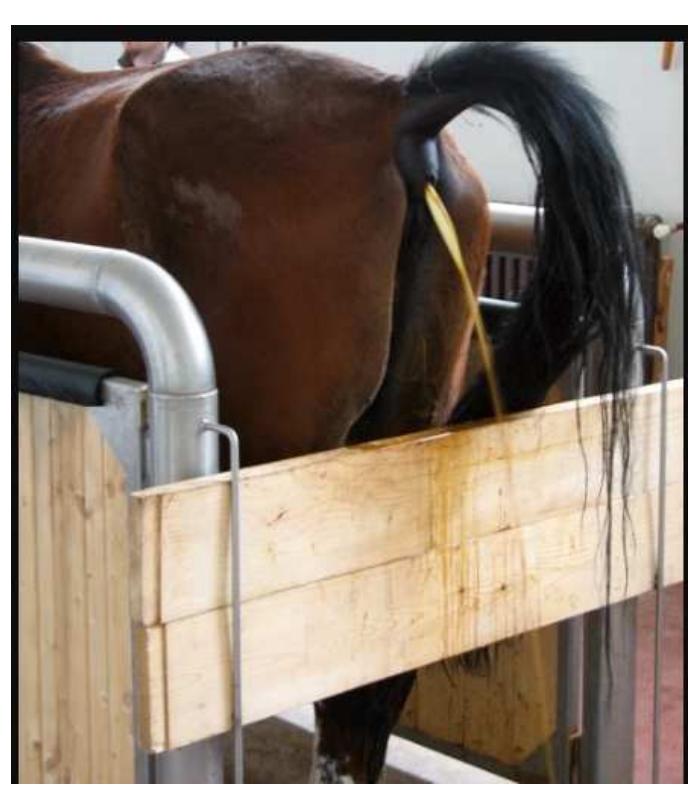

Fig. 1. Profuse diarrhea in an adult horse

In veterinary medicine fecal microbiota transplants have recently received renewed interest, for similar purposes as probiotic products. The efficacy of a fecal microbiota transplant in gastrointestinal disorders in horses is still not supported by scientific evidence and reports of its use in equine medicine are still just anecdotal. Good results of fecal microbiota transplant in other animals and humans have encouraged positive effects expectance in the treatment of acute and chronic diarrhea and inflammatory bowel disease in horses (Mullen et al., 2016).

\section{Clinical Studies in Adult Horses}

Objective clinical studies on probiotic efficacy in horses are scarce in comparison to a diverse range of specialized equine probiotic products available on the market (Weese, 2002b). Most of the microorganisms that can be found in these products have never been subjected to clinical studies and evaluation in horses (Weese and Martin, 2011). Horse owners are usually not informed about the details of such products and it would seem that purchasing an effective product is a matter of chance, given that they have most likely not been subjected to quality control and/or effectiveness testing (Weese et al., 2003; Weese and Martin, 2011).

Moreover, the exact dose required to efficiently colonize the adult equine intestinal tract is still undetermined for the majority of probiotics (Weese, 2003). Most of the studies (Parraga et al., 1997; Kim et al., 2001; Ward et al., 2004; Desrochers et al., 2005) used dosing recommendations given by the product's manufacturer. Assuming that the dosing can be embraced from those used in human medicine, dosing recommendations for horses have been adjusted by weight, although this is not manageable with all commercial probiotic preparations (Weese, 2001). Furthermore, studies using multiple dosage options are rare. An interesting study by Weese et al. (2003) showed better colonization results in foals treated with smaller doses, while adult horses showed proof of colonization only when given high, prohibited probiotic doses. When used in clinical studies it is mandatory to confirm the exact dosing by additional microorganisms viability control (Weese and Martin, 2011).

Since some studies lack basic research principles like blinding, unequality between testing and control groups, unclear randomization, insufficient monitoring of animals and/or used probiotic products, their results should be regarded with caution (Schoster et al., 2015a).

Clinical studies are mainly performed on healthy animals (Medina et al., 2002; Ward et al., 2004; Landes et al., 2008; Tanabe et al., 2014), but it is possible to foresee that probiotic preparations are more often needed for the sick animals. It is than questionable whether the same probiotic organisms have any beneficial effect on gut health at the time of microbiota dysbiosis, or whether they can cause greater damage in these cases (Weese and Rousseau, 2005). Clinical trials on unhealthy animals are far more demanding, although their findings would be of great importance in probiotic testing evaluation.

Nosocomial infections caused by Salmonella shedding led to veterinary hospital closures causing great economic loses (Kim et al., 2001). It is, therefore, of high economic interest to reduce all the possible Salmonella outbreaks. Several clinical trials evaluated potential probiotic effects in hospitalized horses. Parraga et al. (1997) evaluated two different commercial probiotic preparations. One product contained Lactobacillus plantarum, $L$. casei, L. acidophilus and Streptococcus faecium and the other consisted of L. acidophilus, $\mathrm{S}$ faecium, Bifidobacterium thermophilum, B. longum. They were evaluated in 200 adult horses undergoing colic surgery. A more recent study evaluated the effect of one commercial product containing Lactobacillus lactis, Enterococcus faecium, live yeast cells and vitamins on fecal Salmonella shedding and clinical signs of the disease in 246 colic patients (Kim et al., 2001). Results of both studies did not show any effect on fecal shedding of Salmonella, nor did they decrease the likelihood of postoperative diarrhea or shorten the duration of antimicrobial therapy or hospitalization. They assumed inadequate dose or treatment duration could be blamed for the futile results (Parraga et al., 1997). It was proposed that probiotic treatment could show better results in reducing fecal Salmonella shedding if used as a prophylactic treatment, prior to clinical manifestation of the gastrointestinal disease (Kim et al., 2001). A study by Ward et al. (2004) was the only one to show promising results on Salmonella fecal shedding reduction in veterinary teaching hospital. It included 130 horses without signs of 
gastrointestinal disease and 68 of them received an oral gel product containing Lactobacillus plantarum, $L$. casei, L. acidophilus and Enterococcus faecium, reducing the incidence of Salmonella shedding by approximately $65 \%$ in probiotic treated group. The authors suggested that the potential preventive effects of probiotics were better evidenced than in previous studies since they received the probiotic treatment prior, rather than concurrently with antibiotic therapy and enrolled only horses without signs of gastrointestinal disease.

An interesting study on a combined product of prebiotic, probiotic and psyllium showed improved fecal sand clearance in horses (Landes et al., 2008). The probiotic prebiotic product contained Saccharomyces cerevisiae, Lactobacillus acidophilus and Enterococcus faecium combined with $99.5 \%$ whole blond psyllium husk produced into a pellet was administered daily for 35 days. Fecal collection and sand determination were done every day of a 7-day pretreatment period and for the first 14 days and the last 7 days of supplement treatment. An average fecal sand output was at least 2.5 times greater during treatment, compared to the pretreatment period. The results suggest this product is a useful prophylactic treatment against sand enteropathy and sand colic (Landes et al., 2008).

Despite some research have to be regarded with caution, there has been more available research on yeasts than on bacterial impact on equine microbiota and most of them with positive effects on equine gut health. Two studies on Saccharomyces cerevisiae influence on digestion and gut health showed promising results (Medina et al., 2002; Jouany et al., 2008). One study monitored $S$. cerevisiae impact on microbial profiles and fermentation patterns in the large intestine (Medina et al., 2002) and the other was focused on digesta flow rate and apparent digestibility measurements (Jouany et al., 2008). Both studies were done on 8 cecum- and colonfistulated horses, fed a high fiber or a high starch diet. Saccharomyces cerevisiae survived, but failed to colonize large intestine in both studies (Medina et al., 2002; Jouany et al., 2008), although it reduced the decrease in $\mathrm{pH}$ and the after feeding increase in lactic acid in horses fed starchy diets. Furthermore, it also appeared to limit the extent of undesirable changes in the intestinal ecosystem of the horse when the small intestine was saturated with starch (Medina et al., 2002). Saccharomyces cerevisiae supplementation used in both, high starch and high fiber diets, significantly improved cellulose fraction digestibility (Jouany et al., 2008).

A study on Saccharomyces boulardii administration in horses with acute enterocolitis showed significant decrease in the duration of watery diarrhea and the duration of gastrointestinal illness (Desrochers et al., 2005). In this study 7 horses received the treatment and another 7 received placebo and no difference was found between these two groups in hospitalization duration, recurrence of diarrhea during hospitalization or disease outcome. Besides, it is also important to mention that all horses received standard antidiarrheic treatment for acute enterocolitis. A similar trial was conducted on 21 horses with antimicrobial-associated diarrhea (Boyle et al., 2013). It proved the survival of $S$. boulardii in horses with gastrointestinal illness, "demonstrating that it can survive in the gastrointestinal tract of horses with intestinal pathology". However, in contrast to findings by Desrochers et al. (2005), it did not prove that the treatment with $S$. boulardii had any impact on the duration of watery diarrhea.

A double-blinded crossover study on combined product containing $L$. acidophilus and some yeast species, including $S$. cerevisiae and $S$. boulardii, prebiotics and biogenics used in healthy horses, resulted in improved intestinal health (Ishizaka et al., 2014). An oral liquid product of probiotics was administered to 10 horses for 28 days and it increased L. acidophilus and $S$. cerevisiae levels in the gut and diminished the relative amounts of enter pathogenic bacterial DNA. The effect of the probiotic portion of this product could not be separately evaluated since it also contained prebiotics and biogenics (Ishizaka et al., 2014). Prebiotics consisting of short-chain fructooligosaccharides have been reported to be effective in reducing disruptions of the microbial populations in the equine hindgut (Respondek et al., 2008).

The only study focusing on immune system response in horses tested a combined product of two organisms: Pediococcus acidilactici and Saccharomyces boulardii (Furr, 2014). An in vitro testing was followed by a clinical trial on 12 horses. Proliferative responses of cultured PBMCs, serum immunoglobulin concentrations, lymphocyte phenotype subsets, white blood cell count and vaccination response were observed in horses fed a probiotic product for 68 days. Outcomes of the study showed that the used probiotic had an effect on systemic immunity, specifically proliferative responses, immunoglobulin $\mathrm{G}$ concentrations and neutrophil numbers, however, most of the investigated effects did not show statistical significance (Furr, 2014).

A study by Swyers et al. (2008) aimed at determining the effect of direct-fed LAB on digestibility and minimization of the acidosis development in horses whose dietary starch content was abruptly increased. The study showed only limited effects of single (Lactobacillus acidophilus) or mixed strain direct-fed lactic acid bacteria (consisting of Bifidobacterium bifidum, Enterococcus faecium, Lactobacillus acidophilus and Lactobacillus casei) on nutrient digestibility but none prevented acidosis.

\section{Clinical Studies in Foals}

Severe gastrointestinal diseases in neonatal foals are quite common and more than half of foals develop diarrhea in the first six months of life (Frederick et al., 
2009). They are most commonly caused by Clostridium difficile, Clostridium perfringens, Salmonella spp, rotaviral infections, or can be multifactorial, but the etiology of the disease is undetermined in most of the cases (Netherwood et al., 1996; 1998; Frederick et al., 2009). Hence, they represent an interesting research subject for probiotic effectiveness. Carrying out research studies on neonatal foals has great advantages; they are easily managed, simple to equalize in the research groups and the mares mostly foal in the same season of the year. Collecting a similar group of mature horses with the same disease in a short time period is hard to achieve. For example, a study done by Boyle et al. (2013) took over a two-and-a-half-year period in a busy equine veterinary hospital to collect a total of 21 adult horses with diarrhea. Anyhow, the results obtained in research on foals should not be applied to adult horses, due to the immaturity of the digestive tract in young animals (Weese and Rousseau, 2005).

Lactobacillus rhamnosus strain GG, the mostly investigated probiotic strain in human medicine (Goldin et al., 1992), was evaluated by Weese et al. (2003) for its potential of the gastrointestinal tract colonization in horses. The study was done simultaneously in adult horses and foals and showed tremendous differences between mature and immature animals. Very low colonization abilities were shown in adult horses which received exceedingly high doses of the yeast, while foals showed very good response to colonization, lasting up to 9 days after administration of the last probiotic dose. The results concerning dosing in foals turned out opposite from expected, since higher fecal doses were found in the lower dose group of foals (Weese et al., 2003). There were no adverse effects noted in any of the animals, even in the high dose groups (Weese, 2003). The poor adaptation of LGG with the intestinal microflora of adult horses was attributed to its human origin (Weese, 2003). However, the good results achieved with the same bacterial strain in foals do not support this assumption. It is advised, though, to avoid LGG use in foals less than $24 \mathrm{~h}$ old, because there is a possibility of interference with the passive transfer of maternal antibodies, since antigen transport in the intestinal tract causing the large molecular transport pores to close was described (Gorbach, 2000). Weese et al. (2003) concluded that "Lactobacillus rhamnosus GG cannot be considered an equine probiotic at this point" and suggested performing efficacy studies in foals since they showed adequate colonization of LGG in foals.

Tanabe et al. (2014) evaluated an equine origin multistrain probiotic preparation by previously isolating commensals: Four Lactobacillus strains and one Bifidobacterium bacteria. The study evaluated the incidence of diarrhea in neonatal Thoroughbred foals, with a focus on the anti-inflammatory and the intestinal barrier-protective activities. Their results indicated intestinal function regulation and diarrhea prevention in treated foals. However, due to the limitations of the study (lack of blinding, unclear randomization and monitoring of the foals, unequally sized control group and no quality control of the administered probiotic), their results are somewhat questionable (Schoster et al., 2015a).

A study using equine specific five strains Lactobacillus probiotic isolated from healthy horses suggested enhanced growth and decreased incidence of diarrhea in neonatal foals. Increased body weight was noted in foals at 2 to 4 weeks of age and a lower diarrhea incidence in $51.9 \%$ foals 2 to 3 weeks old (Yuyama et al., 2004). Opposite outcomes in neonatal foals were found in another study also using equineorigin organism, Lactobacillus pentosus WE7 (Weese and Rousseau, 2005). This probiotic organism was chosen as "subjectively superior" among 47 other LAB microorganisms isolated from horse manure and was first tested in in vitro conditions and subjected to a preliminary safety trial prior to the clinical study, showing promising beneficial effects in horses (Weese et al., 2004). But instead, the randomized controlled clinical trial on 153 foals showed disappointing results. In fact, rather than preventing gastrointestinal disturbances, it caused diarrhea and even promoted the need for veterinary treatment (Weese and Rousseau, 2005).

The same adverse effects were also presented in a study on clostridial fecal shedding in foals (Schoster et al., 2015a). These studies raised serious concerns regarding the safety of probiotic preparations in foals. Probiotic effect on prevention of diarrhea and Clostridium perfringens and Clostridium difficile fecal shedding was evaluated in 72 healthy foals. Bacterial strains of Lactobacillus rhamnosus, Bifidobacterium animalis lactis and two strains of L. plantarum were administered to foals for 3 weeks and multiple fecal samples were collected. Even though in vitro activity of this probiotic showed good results, there was no influence on Clostridial fecal shedding in this study. The following study by Schoster et al. (2015b) included 38 foals from a previous study investigating the probiotics effects on the bacterial microbiota during and after administration. Insignificant differences in relative abundance of families and species in treated foals proved limited potential of the used probiotics for therapeutic modification of the gastrointestinal microbiota. Lactobacillus- and Bifidobacterium-based probiotics are currently considered ineffective in prevention of diarrhea in neonatal foals (Weese and Rousseau, 2005; Schoster et al., 2015a; 2015b), however further clinical studies on various probiotic microorganisms are needed to evaluate their properties in foals. 


\section{Conclusion}

Reviewing all the results on clinical use of probiotics in adult horses and foals, the benefits of their applicability still remain unclear. The effectiveness of probiotic products in horses has been proven in very few studies. The inability of the large intestine colonization after administration cessation and a demand for daily use do not favor prophylactic properties of probiotics in horses. It is recommended to perform in vitro studies to identify potentially beneficial properties of studied probiotic organisms, prior to evaluation of its' clinical efficiency, nevertheless, available commercial products that lack published safety and efficacy data should be avoided.

The research done is still insufficient to properly evaluate probiotic use in horses, thus more studies, on different microorganisms, are paramount at this point.

\section{Author's Contributions}

Jelena Gotić: Prepared the original draft of this review manuscript.

Darko Grden: Contributed to the contents and checked for English spelling.

Nikica Prvanović Babić: Contributed to the manuscript preparation.

Vladimir Mrljak: Contributed to the review article contents including the design, execution, interpretation and manuscript preparation.

All authors read and approved the final manuscript.

\section{Ethics}

No ethical issues are concerned with the present article.

\section{References}

Al Jassim, R.A. and F.M. Andrews, 2009. The bacterial community of the horse gastrointestinal tract and its relation to fermentative acidosis, laminitis, colic and stomach ulcers. Vet. Clin. N. Am. Equine, 25: 199-215. DOI: 10.1016/j.cveq.2009.04.005

Berg, R.D., 1998. Probiotics, prebiotics or "conbiotics"? Trends Microbiol., 6: 89-92. DOI: 10.1016/S0966-842X(98)01224-4

Bleichner, G., H. Blehaut, H. Mentec and D. Moyse, 1997. Saccharomyces boulardii prevents diarrhea in critically ill tube-fed patients. A multicenter, randomized, double-blind placebo-controlled trial. Intens. Care Med., 23: 517-23. DOI: $10.1007 / \mathrm{s} 001340050367$

Boyle, A.G., K.G. Magdesian, R. Gallop, S. Sigdel and M.M. Durando, 2013. Saccharomyces boulardii viability and efficacy in horses with antimicrobial-induced diarrhoea. Vet. Rec., 172: 128. DOI: $10.1136 /$ vr. 10083392
Cebrian, R., A. Banos, E. Valdivia and M.J. Flores, 2012. Characterization of functional, safety and probiotic properties of Enterococcus faecalis UGRA10, a new AS-48-producer strain. Food Microbiol., 30: 59-67. DOI: 10.1159/000106091

Costa, M.C. and J.S. Weese, 2012. The equine intestinal microbiome. Anim. Health Res. Rev., 13: 121-8. DOI: $10.1017 / \mathrm{S} 1466252312000035$

Costa, M.C., L.G. Arroyo, E. Allen-Vercoe, H.R. Stampfli and P.T. Kim et al., 2012. Comparison of the fecal microbiota of healthy horses and horses with colitis by high throughout sequencing of the V3V5 region of the $16 \mathrm{~S}$ rRNA gene. PLoS ONE. DOI: 10.1371/journal.pone.0041484

Costa, M.C., G. Silva, R.V. Ramos, H.R. Staempfli and L.G. Arroyo et al., 2015a. Characterization and comparison of the bacterial microbiota in different gastrointestinal tract compartments in horses. Vet. J., 205: 74-80. DOI: 10.1016/j.tvj1.2015.03.018

Costa, M.C., H.R. Staempfli, L.G. Arroyo, E. Allen-Vercoe and R.G. Gomes et al., 2015 b. Changes in the equine fecal microbiota associated with the use of systemic antimicrobial drugs. B.M.C. Vet. Res., 11: 19-19. DOI: 10.1186/s12917-015-0335-7

Desrochers, A.M., B.A. Dolente, M.F. Roy, R. Boston and S. Carlisle, 2005. Efficacy of Saccharomyces boulardii for treatment of horses with acute enterocolitis. J. Am. Vet. Med. Assoc., 227: 954-959. DOI: $10.2460 /$ javma.2005.227.954

Francis-Smith, K. and D.G.M. Wood-Gush, 1977. Coprophagia as seen in Thoroughbred Foals. Equine Vet. J., 9: 155-7.

Frederick, J., S. Giguere and L.C. Sanchez, 2009. Infectious agents detected in the feces of diarrheic foals: A retrospective study of 233 cases (20032008). J. Vet. Intern. Med., 23: 1254-1260. DOI: $10.1111 /$ j.1939-1676.2009.0383.x

Fuller, R., 1991. Probiotics in human medicine. Gut, 32: 439-442.

Furr, M., 2014. Orally Administered Pediococcus acidilactici and Saccharomyces boulardii-based probiotics alter select equine immune function parameters. J. Equine Vet. Sci., 34: 1156-1163. DOI: $10.1016 /$ j.jevs.2014.06.020

Garrett, L.A., R. Brown and I.R. Poxton, 2002. A comparative study of the intestinal microbiota of healthy horses and those suffering from equine grass sickness. Vet. Microbiol., 87: 81-8. DOI: 10.1016/S0378-1135(02)00018-4

Gibson, G.R. and R. Fuller, 2000. Aspects of in vitro and in vivo research approaches directed toward identifying probiotics and prebiotics for human use. J. Nutr., 130: 391S-395S. PMID: 10721913

Gorbach, S.L., 2000. Probiotics and gastrointestinal health. Am. J. Gastroenterol., 95: S1-S4. DOI: 10.1016/S0002-9270(99)00806-0 
Goldin, B.R., S.L. Gorbach, M. Saxelin, S. Barakat and L. Gualtier et al., 1992. Survival of Lactobacillus species (Strain GG) in human gastrointestinal tract. Dig. Dis. Sci., 37: 121-128. DOI: $10.1007 / \mathrm{BF} 01308354$

Guandalini, S., L. Pensabene, M.A. Zikri, J.A. Dias and L.G. Casali, 2000. Lactobacillus GG administered in oral rehydration solution to children with acute diarrhea: A multicenter Euopean trial. J. Ped. Gastro. Nutr., 30: 54-60. DOI: 10.1097/00005176-200001000-00018

Gueimonde, M., B. Sanchez, C.G. de los Reyes-Gavilan and A. Margolles, 2013. Antibiotic resistance in probiotic bacteria. Front. Microbiol., 4: 202-202. DOI: $10.3389 /$ fmicb.2013.00202

Gupta, P., H. Andrew, B.S. Kirschner and S. Guandalini, 2000. Is Lactobacillus GG helpful in children in Crohn's disease? Results of a preliminary, openlabel study. J. Ped. Gastro. Nutr., 31: 453-457. DOI: 10.1097/00005176-200010000-00024

Hamilton-Miller, J.M.T. and S. Shah, 2002. Deficiencies in microbiological quality and labelling of probiotic supplements. Int. J. Food Microbiol., 72: 175-176. DOI: $10.1016 / \mathrm{S} 0168-1605(01) 00703-6$

Harlow, B.E., L.M. Lawrence and M.D. Flythe, 2013. Diarrhea- associated pathogens, lactobacilli and cellulolytic bacteria in equine feces: Responses to antibiotic challenge. Vet. Microbiol., 166: 225-232. DOI: 10.1016/j.vetmic.2013.05.003

Isolauri, E., M. Juntunen, T. Pautanen, P. Sillanaukee and T. Koivula, 1991. A human Lactobacillus strain (Lactobacillus casei sp strain GG) promotes recovery from acute diarrhea in children. Pediatrics, 88: 90-97. DOI: 10.1203/00006450-199005000-00026

Ishizaka, S., A. Matsuda, Y. Amagai, K. Oida and H. Jang et al., 2014. Oral administration of fermented probiotics improves the condition of feces in adult horses. J. Equine Sci., 25: 65-72. DOI: $10.1294 /$ jes. 25.65

Jouany, J.P., J. Gobert, B. Medina, G. Bertin and V. Julliand, 2008. Effect of live yeast culture supplementation on apparent digestibility and rate of pas- sage in horses fed a high-fiber or high-starch diet. J. Anim. Sci., 86: 339-347. DOI: 10.2527/jas.2006-796

Kailasapathy, K. and J. Chin, 2000. Survival and therapeutic potential of probiotic organisms with reference to Lactobacillus acidophilus and Bifidobacterium spp. Immunol. Cell Biol., 78: 80-88. DOI: 10.1046/j.1440-1711.2000.00886.x

Kim, L.M., P.S. Morley, J.L. Traub-Dargatz, M.D. Salman and C. Gentry-Weeks, 2001. Factors associated with Salmonella shedding among equine colic patients at a veterinary teaching hospital. J. Am. Vet. Med. Assoc., 218: 740-748. DOI: $10.2460 /$ javma.2001.218.740
Kronfeld, D.S. and P. Harris, 1997. Feeding the Athletic Horse. In: The Veterinarian's Practical Reference to Equine Nutrition. K.N. Thompson (Ed.) Purina Mills, Inc., St. Louis, MO. ISBN-13: 9780965959001 , pp: 61-77.

Kyriakis, S.C., V.K. Tsiloyiannis, J. Vlemmas, K. Sarris and A.C. Tsinas et al., 1999. The effect of probiotic LSP 122 on the control of post-weaning diarrhoea syndrome of piglets. Res. Vet. Sci., 67: 223-228. DOI: $10.1053 /$ rvsc. 1999.0308

Landes, A.D., D.M. Hassel, J.D. Funk and A. Hill, 2008. Fecal sand clearance is enhanced with a product combining probiotics, pre- biotics and psyllium in clinically normal horses. J. Equine Vet. Sci., 28: 79-84. DOI: $10.1016 /$ j.jevs.2008.01.004

Lallès, J.P., P. Bosi, H. Smidt and C.R. Stokes, 2007. Nutritional management of gut health in pigs around weaning. Proc. Nutr. Soc., 66: 260-268. DOI: $10.1017 / \mathrm{S} 0029665107005484$.

Lee, D.J., R.A. Drongowski, A.G., Coran and C.M. Harmon, 2000. Evaluation of probiotic treatment in a neonatal animal model. Pediatr. Surg. Int., 6: 237-242. DOI: 10.1007/s003830050736.

Lilley, D.M. and R.H. Stillwell, 1965. Probiotics: Growth promoting factors produced by microorganisms. Science, 147: 747-8.

Lund, B. and C. Edlund, 2001. Probiotic Enterococcus faecium strain is a possible recipient of the van A gene cluster. Clin. Infect. Dis., 32 : 1384-1385. DOI: $10.1086 / 319994$

Mater, D.D., P. Langella, G. Corthier and M.J. Flores, 2008. A probiotic Lactobacillus strain can acquire vancomycin resistance during digestive transit in mice. J. Mol. Microbiol. Biotechnol., 14: 123-127. DOI: $10.1159 / 000106091$

Medina, B., I.D. Girard, E. Jacotot and V. Julliand, 2002. Effect of a preparation of Saccharomyces cerevisiae on microbial profiles and fermentation patterns in the large intestine of horses fed a high fiber or a high starch diet. J. Anim. Sci., 80: 2600-2609. DOI: $10.2527 / 2002.80102600 \mathrm{x}$

Metchnikoff, E., 1908. Lactic Acid as Inhibiting Intestinal Putrefaction. In: The Prolongation of Life: Optimistic Studies, Mitchell, P.C. (Ed), Putnam, London, pp: 116-129.

Mullen, K.R., K., Yasuda, T.J. Divers and J.S. Weese, 2016. Equine faecal microbiota transplant: Current knowledge, proposed guidelines and future directions. Equine Vet. Educ. DOI: 10.1111/eve.12559

Netherwood, T., J.L. Wood, H.G. Townsend and J.A. Mumford, 1996. Foal diarrhoea between 1991 and 1994 in the United Kingdom associated with Clostridium perfringens, rotavirus, Strongyloides westeri and Cryptosporidium spp. Epidemiol. Infect., 117: 375-383. DOI: $10.1017 / \mathrm{S} 0950268800001564$ 
Netherwood, T., M. Binns, H. Townsend, J.L.N. Wood and J.A. Mumford et al., 1998. The Clostridium perfringens enterotoxin from equine isolates: Its charac- terization, sequence and role in foal diarrhoea. Epidemiol. Infect., 120: 193-200. PMID: 9593490

Parraga, M.E., S.J. Spier, M. Thurmond and D. Hirsh, 1997. A clinical trial of probiotic administration for prevention of Salmonella shedding in the postoperative period in horses with colic. J .Vet. Intern. Med., 11: 36-41. DOI: 10.1111/j.1939-1676.1997.tb00071.x

Reid, G., 2000. In defense of probiotics. Am. Soc. Microbiol. News, 66: 261-261.

Respondek, F., A.G. Goachet and V. Julliand, 2008. Effects of dietary short-chain fructooligosaccharides on the intestinal microflora of horses subjected to a sudden change in diet. J. Anim. Sci., 86: 316-323. DOI: $10.2527 /$ jas.2006-782

Rinkinen, M., K. Jalava, E. Westermarck, S. Salminen and A.C. Ouwehand, 2003. Interaction between probiotic lactic acid bacteria and canine enteric pathogens: A risk factor for intestinal Enterococcus faecium colonization. Vet. Microbiol., 92: 111-119. DOI: $10.1016 / \mathrm{S} 0378-1135(02) 00356-5$

Saarela, M., G. Mogensen, R. Fonden, J. Mättö and T. Mattila-Sandholm, 2000. Probiotic bacteria: Safety, functional and technological properties. J. Biotech., 84: 197-215. DOI: 10.1016/S0168-1656(00)00375-8

Saavedra, J., 2000. Probiotics and infectious diarrhea. Am. J. Gastroenterol., 95: S16-S18.

Schlundt, J., 2014. Health and nutritional properties of probiotics in food including powder milk with live lactic acid bacteria-Joint FAO/WHO expert consultation. Eval. Health Nutr. Properties Probiotics Food Including Powder Milk Live Lactic Acid Bact. FAO/ WHO.

Schoster, A., L.G. Arroyo, H.R. Staempfli and J.S. Weese, 2013. Comparison of microbial populations in the small intestine, large intestine and feces of healthy horses using terminal restriction fragment length polymorphism. BMC Res. Notes, 6: 91-91. DOI: 10.1186/1756-0500-6-91

Schoster, A., J.S. Weese and L. Guardabassi, 2014. Probiotic use in horses-what is the evidence for their clinical efficacy? J. Vet. Intern. Med., 28: 1640-1652. DOI: 10.1111 /jvim. 12451

Schoster, A., H.R. Staempfli, M. Abrahams, M. Jalali and J.S. Weese et al., 2015a. Effect of a probiotic on prevention of diarrhea and Clostridium difficile and Clostridium perfringens shedding in foals. J. Vet. Intern. Med., 29: 925-931. DOI: 10.1111/jvim. 12584

Schoster, A., L. Guardabassi, H.R. Staempfli, M. Abrahams and M. Jalali et al., 2015b. The longitudinal effect of a multi-strain probiotic on the intestinal bacterial microbiota of neonatal foals. Equine Vet. J. DOI: 10.1111/evj.12524
Swyers, K.L., A.O., Burk, T.G. Hartsock, E.M. Ungerfeld and J.L., Shelton. 2008. Effects of direct-fed microbial supplementation on digestibility and fermentation endproducts in horses fed low-and highstarch concentrates. J. Anim. Sci., 86: 2596-2608. DOI: $10.2527 /$ jas.2007-0608

Tanabe, S., T. Suzuki, Y. Wasano, F. Nakajima and H. Kawasaki et al., 2014. Anti-inflammatory and intestinal barrier-protective activities of commensal lactobacilli and bifidobacteria in Thoroughbreds: role of probiotics in diarrhea prevention in neonatal Thoroughbreds. J. Equine Sci., 25: 37-43. DOI: 10.1294/jes.25.37

Van Immerseel, F., J.B. Russell, M.D. Flythe, I. Gantois and L. Timbermont et al., 2006. The use of organic acids to combat Salmonella in poultry: A mechanistic explanation of the efficacy. Avian Pathol., 35: 182-188. DOI: $10.1080 / 03079450600711045$

Ward, M.P., C.A. Alinovi, L.L. Couetil. T. Glickman and C.C. $\mathrm{Wu}, 2004$. A randomized clinical trial using probiotics to prevent Salmonella fecal shedding in hospitalized horses. J. Equine Vet. Sci., 24: 242-247. DOI: 10.1016/j.jevs.2004.05.004

Weese, J.S., 2001. A review of probiotics: Are they really "functional foods". AAEP Proc., 47: 27-31.

Weese, J.S., 2002a. Probiotics, prebiotics and synbiotics. J. Equine Vet. Sci., 22: 357-360. DOI: $10.1016 / \mathrm{S} 0737-0806(02) 70006-3$

Weese, J.S., 2002b. Microbiologic evaluation of commercial probiotics. J. Am. Vet. Med. Assoc., 220: 794-797. DOI: 10.2460/javma.2002.220.794

Weese, J.S., M.E. Anderson, A. Lowe and G.J. Monteith, 2003. Preliminary investigation of the probiotic potential of Lactobacillus rhamnosus strain GG in horses: Fecal recovery following oral administration and safety. Can. Vet. J., 44: 299-302. PMID: 12715981

Weese, J.S., 2003. Evaluation of deficiencies in labeling of commercial probiotics. Can. Vet. J., 44: 982-983.

Weese, J.S., M.E.C. Anderson, A. Lowe, R. Penno and T.M. Da Costa et al., 2004. Screening of the equine intestinal microflora for potential probiotic organisms. Equine Vet. J., 36: 351-355. DOI: $10.2746 / 0425164044890616$

Weese, J.S. and J. Rousseau, 2005. Evaluation of Lactobacillus pentosus WE7 for prevention of diarrhea in neonatal foals. J. Am. Vet. Med. Assoc., 226: 2031-4. DOI: 10.2460/javma.2005.226.2031

Weese, J.S. and H. Martin, 2011. Assessment of commercial probiotic bacterial contents and label accuracy. Can. Vet. J., 52: 43-46. PMID: 21461205

Wynn, S.G., 2009. Probiotics in veterinary practice. J. Am. Vet. Med. Assoc., 234: 606-613.

Yuyama, T., S. Yusa, S. Takai, S. Tsubaki and Y. Kado et al., 2004. Evaluation of a host-specific Lactobacillus probiotic in neonatal foals. J. Applied Res. Vet. Med., 2: 26-32. 
Zbinden, R., E. Bonczi and M. Altwegg, 1999. Inhibition of Saccharomyces boulardii on cell invasion of Salmonella typhimurium and Yersinia enterocolitica. Microb. Ecol. Health D., 11: 158-162.

DOI: $10.1080 / 089106099435736$
Zhao, T., M.P. Doyle, B.G. Harmon, C.A. Brown and P.O.E. Mueller et al., 1998. Reduction of carriage of enterohemorrhagic Escherichia coli O157:H7 in cattle by inoculation with probiotic bacteria. J. Clin. Microbiol., 36: 641-647. PMID: 9508288 